\title{
OLIGOPOLY EQUILIBRIUM IN PURE EXCHANGE ECONOMIES
}

\author{
JEAN J. GABSZEWICZ \\ CORE, Université Catholique de Louvain \\ Louvain-la-Neuve, Belgium \\ E-mail: gabszewicz@core.ucl.ac.be
}

1. The Cournotian assumptions. The purpose of this paper is to propose and discuss a concept of oligopoly equilibrium in the context of a pure exchange economy ${ }^{1}$. It may seem strange a priori to evoke oligopoly theory in this context, so true is it that economists have always analysed the consequences of market power in the framework of productive activities and partial analysis. However the context of exchange constitutes the natural setting in which to initiate the modeling of "competition among the few" when it takes place in a general equilibrium framework. Not only the same step was taken at the start in order to analyse general equilibrium under pure competition (Wald, 1936; Debreu and Scarf, 1963), but it also allows to discard several conceptual difficulties which are met when oligopoly is embedded in a general equilibrium model with production. Nevertheless we try to remain as close as possible to the basic building blocks underlying the Cournotian analysis of oligopoly in the partial equilibrium context.

The first among these building blocks consists in assuming the existence of a continuous and decreasing demand function $p(Q)$, expressing the price $p(Q)$ at which the quantity $Q$ of a good is sold on its market: Cournot (1838) called this function "la loi de débit". This demand function was meant to agregate the individual demands, at each price, of a large number of competitive buyers, with varying willingness to pay for a unit of that good. On the supply side, Cournot assumes the existence of $n$ sellers, with $q_{i}$ denoting the quantity supplied by seller $i, i=1, \ldots, n, Q=\sum_{i=1}^{n} q_{i}$. Sellers are only interested in revenue expressed in units of a given numeraire while buyers, "hidden" behind the demand function, are also interested in the consumption of the good exchanged against the numeraire. We call this first assumption the partial equilibrium assumption. The second Cournotian assumption is called the asymmetry assumption. It says that buyers are numerous and behave competitively as price-takers; on the contrary, sellers (oligopolists) are

The paper is in final form and no version of it will be published elsewhere.

${ }^{1}$ This concept has been introduced in Gabszewicz (2002). 
assumed to be few and to behave strategically because they perceive how their individual supply influences the equilibrium market price. The third assumption says precisely that the price at which transactions take place makes the sum $Q=\sum_{i=1}^{n} q_{i}$ of the quantities supplied by the oligopolists equal to consumers' demand at that price: this is the equilibrium price assumption. Finally, Cournot assumes that the oligopolists are prevented to coordinate their supply decisions. In the game theory terminology, these supplies form a Nash equilibrium in the normal-form game obtained by considering the oligopolists as players, quantities $q_{i}$ as strategies, and revenues $q_{i} \cdot p\left(\sum_{k=1}^{n} q_{k}\right), i=1, \ldots, n$, as payoffs (non coordination assumption).

In order to illustrate the above assumptions, consider the following example. Let

$$
p(Q)=1-Q
$$

be the market demand function for some commodity and suppose there are $n$ sellers of the same commodity, all sellers producing the good at zero cost. Then the revenue function $\Pi_{k}\left(q_{k}, \sum_{i=1, i \neq k}^{n} q_{i}\right)$ of seller $k$ is

$$
\Pi_{k}\left(q_{k}, \sum_{i=1, i \neq k}^{n} q_{i}\right)=\left(1-q_{k}-\sum_{i=1, i \neq k}^{n} q_{i}\right) \cdot q_{k} .
$$

Given the quantities $q_{i}, i \neq k$, the revenue $\Pi_{k}$ is maximal when

$$
\frac{\partial \Pi_{k}}{\partial q_{k}}=1-2 q_{k}-\sum_{i=1, i \neq k}^{n} q_{i}=0,
$$

which gives rise to the system of equations

$$
q_{k}=\frac{1-\sum_{i=1, i \neq k}^{n} q_{i}}{2}, \quad k=1, \ldots, n .
$$

From this system, it is easy to see that the only Nash equilibrium is given by

$$
q_{k}^{*}=\frac{1}{n+1}, \quad k=1, \ldots, n .
$$

The competitive solution corresponding to this example is given by the equilibrium price $p_{c}=0$ and the agregate quantity $Q_{c}=1$. Thus, this example points out to an interesting property of the Cournot equilibrium in partial analysis: when the number of sellers tends to infinity, the agregate quantity supplied in the market tends to the competitive supply. We shall come back later to this property in the general equilibrium framework.

2. Multilateral exchange economies with the asymmetry assumption. In order to extend the Cournotian concept of equilibrium to a system of interrelated markets, we introduce formally the well known concept of an exchange economy. By definition, an exchange economy consists of a set of commodities $j, j=1, \ldots, m$, a list of traders $i, i=1, \ldots, n$, with continuous utility functions $u_{i}(x), x \in R_{+}^{m}$, and initial endowments $w_{i}, w_{i} \in R_{+}^{m}$. An allocation is an $n$-tuple of vectors $x_{i}, x_{i} \in R_{+}^{m}$, such that

$$
\sum_{i=1}^{n} x_{i}=\sum_{i=1}^{n} w_{i}
$$


A price system is a vector $p \in R_{+}^{m}$. A competitive allocation is an allocation $\left(x_{1}, \ldots, x_{n}\right)$ for which there exists a price system $p$ such that, for all $i, i=1, \ldots, n$, the vector $x_{i}$ maximises $u_{i}(x)$ under the constraint $p . x \leq p . w_{i}$.

Among the assumptions underlying the Cournotian proposal, the partial equilibrium assumption is of course by essence incompatible with the analysis of multilateral exchange. Thus we have necessarily to abandon this assumption; with this respect, it is useful to consider the following example as an introductory device to move from the partial to the general equilibrium context. This example is borrowed from Codognato and Gabszewicz (1991). The exchange economy consists of $n+2$ traders and two goods. All traders, split into two types, share however the same utility function $u(x)$, defined by

$$
u(x)=u\left(x^{1}, x^{2}\right)=x^{1} x^{2},
$$

while their initial endowments are given by

$$
w_{i}= \begin{cases}\left(\frac{1}{2}, 0\right), & i=1,2, \\ \left(0, \frac{1}{n}\right), & i=3, \ldots, n+2 .\end{cases}
$$

We normalise the price of good 2 by setting it equal to 1 . The individual demand function $x_{i}(p)$, with $p$ denoting the price of good 1 , for traders $i=3, \ldots, n+2$, is easily derived from utility maximisation under the budget constraint $p x^{1}+x^{2}=\frac{1}{n}$, namely

$$
x_{i}(p)=\left(\frac{1}{2 p n}, \frac{1}{2 n}\right) .
$$

If traders of type 1 (traders 1 and 2) would behave as price-takers, taking the exchange rate $p$ between goods 1 and 2 as given, their individual demand for good 1 would be each equal to $\frac{1}{4}$, irrespective of the value of $p$. Accordingly, aggregate demand for good 1 at price $p$ would then be equal to $\frac{1}{2}+\frac{1}{2 p}$, so that the aggregate demand for this good would be equal to aggregate supply, which is equal to 1 , if and only if the price $p^{*}$ is equal to 1 . Then the resulting competitive allocation is

$$
x_{i}^{*}= \begin{cases}\left(\frac{1}{4}, \frac{1}{4}\right), & i=1,2, \\ \left(\frac{1}{2 n}, \frac{1}{2 n}\right), & i=3, \ldots, n+2 .\end{cases}
$$

Assume now that the number $n$ is very large so that each trader of type 2 appears as "negligible" in the sense that he only owns a rather insignificant share of the total market endowment of good 2. On the contrary, traders 1 and 2 are still keeping a significant share of the total market endowment in good 1. Thus it is natural to assume that traders of type 2 behave competitively, while the two owners of good 1 want to manipulate at their advantage the exchange rate between the two commodities. Accordingly, this situation evokes, in the context of multilateral exchange, the situation considered by Cournot in partial analysis under the assumption of asymmetry, and spontaneously invites to transpose his equilibrium concept to cover the multilateral exchange context. First observe that the agregate demand for good 1 of the "small" traders $i=3, \ldots, n+2$ 
obtains as $\sum_{i=3}^{n+2} x_{i}^{2}(p)=\frac{1}{2 p}$. Now it is easy to see how the "duopolists" $i=1,2$ can manipulate the equilibrium exchange rate $p$ by restricting their supply of good 1 to any amount $y_{i}$ which is smaller or equal to their initial endowment of this good, namely $y_{i} \in\left[0, \frac{1}{2}\right]$, exactly as the Cournotian oligopolists restrict their market supply -compared with the competitive supply in order to increase the equilibrium market price expressed in the units of the numeraire commodity. Now consider duopolist $i, i=1,2$. If he sends a share $y_{i} \in\left[0, \frac{1}{2}\right]$ of his initial endowment in good 1 to the market for this good, he obtains in exchange a quantity $p y_{i}$ of good 2 when the exchange rate between good 1 and good 2 is equal to $p$. Consequently, using the supply strategy $y_{i}$, duopolist $i$ ends up with the bundle $\left(\frac{1}{2}-y_{i}, p y_{i}\right)$ and reaches the utility level

$$
u\left(\frac{1}{2}-y_{i}, p y_{i}\right)=\left(\frac{1}{2}-y_{i}\right)\left(p y_{i}\right)
$$

But, as in Cournot, duopolist $i$ has a partial control over the equilibrium market price $p$ since, by the equilibrium price assumption, this price must satisfy the market clearing condition

$$
\frac{1}{2 p}=y_{1}+y_{2}
$$

or

$$
p\left(y_{1}, y_{2}\right)=\frac{1}{y_{1}+y_{2}} .
$$

Substituting (2) into (1), we obtain the payoff $V_{i}$ of oligopolist $i$ when he uses the strategy $y_{i}$ and his rival the strategy $y_{k}, k \neq i$, namely

$$
V_{i}\left(y_{1}, y_{2}\right)=\left(\frac{1}{2}-y_{i}\right) \cdot \frac{y_{i}}{2\left(y_{1}+y_{2}\right)} \text {. }
$$

To determine the quantity which is optimal for oligopolist $i$ to send to the market, it is then sufficient to maximise $V_{i}$, given the quantity $y_{k}, k \neq i$, supplied by his rival. Solving the problem for $i=1$, we obtain the first order necessary condition

$$
y_{2}-2 y_{1}^{2}-4 y_{1} y_{2}=0 \text {. }
$$

Similarly, for $i=2$, we get

$$
y_{2}-2 y_{2}^{2}-4 y_{1} y_{2}=0 .
$$

Applying the Cournot non coordination assumption, we get the Cournot equilibrium $\left(y_{1}^{\circ}, y_{2}^{\circ}\right)=\left(\frac{1}{6}, \frac{1}{6}\right)$ from which follows the equilibrium exchange price $p\left(y_{1}^{\circ}, y_{2}^{\circ}\right)=\frac{3}{2}$ and the resulting allocation of goods

$$
x_{i}^{\circ}= \begin{cases}\left(1-y_{i}^{\circ}, p^{\circ} y_{i}^{\circ}\right)=\left(\frac{1}{3}, \frac{1}{4}\right), & i=1,2, \\ \left(\frac{1}{3 n}, \frac{1}{2 n}\right), & i=3, \ldots, n+2 .\end{cases}
$$

To conclude the presentation of this example, the following two remarks are in order. First, when we compare the allocation which we have just described to the competitive allocation, we notice that the duopolists have increased their utility compared with the utility obtained at the latter. This follows from the supply contraction of good 1 which raises the exchange rate between good 1 and good 2 which is equal to $\frac{3}{2}$ at the Cournot 
equilibrium while it is equal to 1 at the competitive equilibrium. Consequently we notice that, as in the Cournotian partial equilibrium approach, agents endowed with market power contract their output supply in order to increase its relative value. Second, it is possible to extend the above example to a situation of oligopoly by replicating the basic exchange economy "à la Debreu-Scarf" when they studied the asymptotic behavior of the core (see Debreu and Scarf, 1963). To this end we define the economy $E_{m}$ as the $m^{\text {th }}$ replica of the economy considered above, say $E_{1}$, namely, the economy $E_{m}$ includes $m(n+2)$ agents, with all agents sharing the same utility function $u\left(x_{1}, x_{2}\right)=x_{1} x_{2}$. Among them $2 m$ agents (the oligopolists) have as initial endowment $w_{1 j}=w_{2 j}=\left(\frac{1}{2}, 0\right)$, $j=1, \ldots, m$, while the $m n$ agents of type 2 have an initial endowment $w_{i j}=\left(0, \frac{1}{n}\right)$, $j=1, \ldots, m ; i=3, \ldots, n+2$. Using the first order necessary and sufficient conditions which are to be satisfied at a Cournot equilibrium of the economy $E_{m}$, we find that this equilibrium leads to a corresponding equilibrium price $p^{\circ}(m)$ given by

$$
p^{\circ}(m)=\frac{2 m-1}{2(4 m-1)}
$$

and the corresponding allocation

$$
x_{i j}^{\circ}(m)= \begin{cases}\left(\frac{m}{4 m-1} \frac{1}{4}\right), & i=1,2, j=1, \ldots, m, \\ \left(\frac{2 m-1}{n(4 m-1)}, \frac{1}{2 n}\right), & i=1, \ldots, n ; j=1, \ldots, m .\end{cases}
$$

Now considering the sequence of allocations $\left\{x_{i j}^{\circ}(m)\right\}$ and the sequence of corresponding equilibrium prices $\left\{p^{\circ}(m)\right\}$, we observe that they converge, respectively, to the competitive allocation and the competitive price in the economy $E_{1}$ when $m \rightarrow \infty$. Thus we conclude that, in this example, as in the example previously presented in the partial equilibrium context, the agregate quantity supplied by the oligopolists tends to increase without limit, causing the resulting prices and allocations to converge to the competitive equilibrium. Thus the treatment of the above example, even if it encompasses only two goods, reveals that there is no difficulty to cast the Cournot theory into a general equilibrium framework, with a closed model, and avoiding to depend on implicit considerations of the type "all other things being equal" which makes partial analysis so vulnerable. Moreover, the above example also shows that the most popular property of the Cournot solution-its convergence to the competitive outcome when the number of oligopolists increases - may also appear as an expected outcome in the general equilibrium framework. The next example shows that this framework has another significant advantage: it also allows to dispense with the awkward assumption of asymmetry.

3. Multilateral exchange economies without the asymmetry assumption. The asymmetry assumption introduced by Cournot in the partial equilibrium model was justified, at least partially, because in several markets, while the number of sellers is rather small, buyers are many. Nevertheless, there are some products for which both sides of the markets embody few sellers only, like in bilateral oligopolies. Furthermore, in the framework of multilateral exchange, in which goods are exchanged for goods, the same agents are simultaneously buyers on some markets and sellers on others. In such contexts, there 
is no reason why strategic consciousness should be assumed for the agents belonging to one particular side of the market: all agents should a priori be treated symmetrically and assume to adopt a strategic behaviour.

Consider then the following alternative version of the example of section 2 . There are now $r+n$ traders with initial endowments

$$
w_{i}= \begin{cases}\left(\frac{1}{r}, 0\right), & i=1, \ldots, r \\ \left(0, \frac{1}{n}\right), & i=r+1, \ldots, r+n .\end{cases}
$$

Again we suppose that all agents share the same utility function $u\left(x^{1}, x^{2}\right)=x^{1} x^{2}$. (Notice that this economy coincides with $E_{1}$ when $r=2$ and we normalise the price of good 2 to be equal to one.) Assume now that all agents in this economy behave strategically, with strategies $y_{i} \in\left[0, \frac{1}{r}\right], i=1, \ldots, r$ and $r_{i} \in\left[0, \frac{1}{n}\right], i=r+1, \ldots, r+n$. For an agent $i=1, \ldots, r$, he obtains in exchange of $y_{i}$ a quantity $p y_{i}$ of good 2. Similarly, for an agent $i=r+1, \ldots, r+n$, he obtains in exchange of $z_{i}$ a quantity $\frac{z_{i}}{p}$ of good 1 . Consequently, at a given $(r+n)$-vector of strategies $\left(y_{1}, \ldots, y_{n} ; z_{r+1}, \ldots z_{r+n}\right)$, payoffs now obtain as

$$
V_{i}\left(y_{1}, \ldots y_{n} ; z_{r+1}, \ldots z_{r+n}\right)= \begin{cases}\left(\frac{1}{r}-y_{i}\right) p y_{i}, & i=1, \ldots, r \\ \frac{z_{i}}{p}\left(\frac{1}{n}-z_{i}\right), & i=r+1, \ldots, r+n .\end{cases}
$$

Keeping the Cournotian equilibrium price assumption, we get from the market clearing condition on the market of good 1 ,

$$
\frac{1}{p} \sum_{k=r+1}^{r+n} z_{k}=\sum_{k=1}^{r} y_{k} ;
$$

or

$$
p=\frac{\sum_{k=r+1}^{r+n} z_{k}}{\sum_{k=1}^{r} y_{k}} .
$$

Substituting in the $V_{i}$ 's, we get the desired payoffs, and easily compute the unique Nash equilibrium

$$
\begin{aligned}
& y_{i}^{\circ}=\frac{r-1}{r(2 r-1)}, i=1, \ldots, r ; \\
& z_{i}^{\circ}=\frac{n-1}{n(2 n-1)}, \quad i=r+1, \ldots, r+n,
\end{aligned}
$$

with equilibrium price $p^{\circ}=\frac{(n-1)(2 r-1)}{(r-1)(2 n-1)}$ and corresponding equilibrium outcomes. First we observe that, keeping $r=2$ and letting the number $n$ tend to $+\infty$, the equilibrium price $p^{\circ}(n)$ tends to the same value $\frac{3}{2}$ as the one obtained in the preceding section with 2 duopolists and n "small" traders behaving as price takers. Even if type-2 agents behave here as strategic agents, they progressively loose their market power because the ownership of good 2 is spread over a larger and larger number of traders while the ownership of good 1 remains concentrated over the duopolists. This should be contrasted with the limit obtained when both $r$ and $n$ tend simultaneously to $+\infty$. In that case, $p^{\circ}(n)$ converges 
to 1 , which corresponds to the exchange rate at the competitive equilibrium: when the number of oligopolists on each side of the bilateral market is simultaneously increased, the oligopoly outcome now converges to the competitive equilibrium. In the next section, we extend the definition of an oligopoly equilibrium to an exchange economy with an arbitrary number of traders and goods.

\section{A formal definition of oligopoly equilibrium and the existence problem.}

Consider an exchange economy with $m$ goods $h, h=1, \ldots, m$ and $n$ agents $i, i=1, \ldots, n$, with utility functions $u_{i}(x)$ and initial endowments $w_{i}, w_{i} \in R_{+}^{m}$. We define the following game. The players are the traders $i, i=1, \ldots, n$. The strategy set $S_{i}$ of player $i$ is defined as $\left\{y \in R_{+}^{m} ; 0 \leq y \leq w_{i}\right\}$. The payoffs are defined as follows. Let $\left(y_{1}, \ldots, y_{i}, \ldots, y_{n}\right)$ be an $n$-tuple of strategies and consider player $i$. Given a price system $p$, let $x_{i}\left(p, y_{i}\right)$ denote the solution to the problem

$$
\operatorname{Max}_{x} u_{i}\left(w_{i}-y_{i}+x\right) \quad \text { s.t. } \quad p . x \leq p . y_{i}^{2} ; x \geq 0 .
$$

Let $p\left(y_{1}, \ldots, y_{i}, \ldots, y_{n}\right)$ denote the price system which solves

$$
\sum_{i=1}^{n} x_{i}\left(p, y_{i}\right)=\sum_{i=1}^{n} y_{i}{ }^{3}
$$

and define $V_{i}$ by

$$
V_{i}\left(y_{1}, \ldots, y_{i}, \ldots, y_{n}\right)=V_{i}\left(y, ; y_{-i}\right)=u_{i}\left(w_{i}-y_{i}+x_{i}\left(p\left(y_{1}, \ldots, y_{i}, \ldots, y_{n}\right), y_{i}\right)\right):
$$

$V_{i}$ is the payoff function of player $i$. Notice however that in the particular case in which, for all $i, i=1, \ldots, n, y_{i}=0$, no trade can occur since no agent has sent a positive amount of any good to the markets for trade. Then each agent necessarily ends up with his initial endowment and consumes as "in autarky". Consequently the corresponding payoff $V_{i}$ is given by $V_{i}(0, \ldots, 0)=u_{i}\left(w_{i}\right)$. Finally we define an oligopoly equilibrium as an $n$-tuple $\left(y_{1}^{\circ}, \ldots, y_{i}^{\circ}, \ldots, y_{n}^{\circ}\right), y_{i}^{\circ} \in S_{i}$, such that, for all $i$ and for all $y_{i} \in S_{i}$,

$$
V_{i}\left(y_{i}, y_{-i}^{\circ}\right) \leq V_{i}\left(y_{i}^{\circ}, y_{-i}^{\circ}\right) \text {. }
$$

The first natural question to raise with respect to the concept of oligopoly equilibrium concerns its existence: does an oligopoly equilibrium exist? When defining the payoffs of the game, we have explicitly considered the possibility that all traders might use simultaneously their "no-trade strategy" $y_{i}=0$. It turns out that the corresponding vector of strategies is always an oligopoly equilibrium: intuitively, it is clear that, if no trader $k, k \neq i$, supplies the market with any amount of any good, it is optimal as well for trader $i$ to supply nothing. This is exactly what is required at a Nash equilibrium: no unilateral deviation from the strategy chosen by each player pays a larger amount of utility. This proves that the $n$-tuple of strategies $(0, \ldots, 0)$ is an oligopoly equilibrium; we call it the trivial equilibrium. This simple reasoning reveals that there always exists

\footnotetext{
${ }^{2}$ Monotonicity, continuity and strict quasi-concavity of $u_{i}$ are sufficient conditions to guarantee the existence of a unique solution to this problem.

${ }^{3}$ Standard assumptions guarantee the existence of a price system $p\left(y_{1}, \ldots, y_{i}, \ldots, y_{n}\right)$ for any $n$-tuple of strategies $\left(y_{1}, \ldots, y_{i}, \ldots, y_{n}\right), y_{i} \in S_{i}, i=1, \ldots, n$; we shall assume hereafter that this price system is unique.
} 
at least one oligopoly equilibrium, the one at which no trade occurs. Furthermore, it is easy to see that there are at least two circumstances in which the trivial equilibrium is the unique one. The reader can easily check by himself that the first corresponds to the case of an exchange economy with two commodities and two traders; and the second when the initial allocation of goods is pareto optimal. The existence problem (for a non trivial equilibrium) must accordingly be reformulated in the following way: does there exist a non-trivial equilibrium for all exchange economies with non-Pareto optimal initial allocations and including more than two traders? Unfortunately, as shown by Cordella and Gabszewicz, 1998, the answer to this question is negative. Consider an exchange economy consisting of $n$ traders, falling into two types, with $\frac{n}{2}$ of each type; there are two goods and we normalise the price of good 2 to be equal to one. For traders $i, i=1, \ldots, \frac{n}{2}$, preferences and endowments are defined by

$$
u_{i}\left(x^{1}, x^{2}\right)=\beta x^{1}+x^{2} ; \quad w_{i}=(1,0) ;
$$

similarly, for traders $i, i=\frac{n}{2}+1, \ldots, n$, we have

$$
u_{i}\left(x^{1}, x^{2}\right)=x^{1}+\beta x^{2} ; \quad w_{i}=(0,1)
$$

with $0<\beta<1$. This exchange economy has a unique competitive equilibrium given by $p^{*}=(1,1)$ and the allocation

$$
x_{i}^{*}= \begin{cases}(0,1), & i=1, \ldots, n / 2, \\ (1,0), & i=n / 2+1, \ldots, n .\end{cases}
$$

Now consider the associated game with strategy sets defined by

$$
S_{i}=\left\{\left(y_{i}^{1}, y_{i}^{2}\right): 0 \leq y_{i}^{1} \leq 1 ; y_{i}^{2}=0\right\}
$$

for $i=1, \ldots, n$ and by

$$
S_{i}=\left\{\left(z_{i}^{1}, z_{i}^{2}\right): z_{i}^{1}=0 ; 0 \leq z_{i}^{2} \leq 1\right\}
$$

for $i=\frac{n}{2}+1, \ldots, n$. Given an $n$-tuple of strategies with $\left(y_{i}^{1}, y_{i}^{2}\right) \in S_{i}, i=1, \ldots, \frac{n}{2}$, and $\left(z_{i}^{1}, z_{i}^{2}\right) \in S_{i}, i=\frac{n}{2}+1, \ldots, n$, the price of $\operatorname{good} 1$ is

$$
p=\frac{\sum_{k=\frac{n}{2}+1}^{n} z_{k}^{2}}{\sum_{k=1}^{\frac{n}{2}} y_{k}^{1}},
$$

with corresponding payoffs

$$
V_{i}\left(y_{i}^{1} ; y_{i}^{2}\right)=\beta\left(1-y_{i}^{1}\right)+y_{i}^{1} \frac{\sum_{k=\frac{n}{2}+1}^{n} z_{k}^{2}}{\sum_{k=1}^{\frac{n}{2}} y_{k}^{1}}
$$

for $i=1, \ldots, \frac{n}{2}$, and

$$
V_{i}\left(z_{i}^{1} ; z_{i}^{2}\right)=z_{i}^{2} \frac{\sum_{k=1}^{\frac{n}{2}} y_{k}^{1}}{\sum_{k=\frac{n}{2}+1}^{n} z_{k}^{2}}+\beta\left(1-z_{i}^{1}\right)
$$

for $i=\frac{n}{2}+1, \ldots, n$. Using first order necessary and sufficient conditions, it is easy to show that

$$
\frac{\partial V_{i}}{\partial y_{i}^{1}} \geq 0 \Leftrightarrow \beta \leq \frac{n-2}{n}
$$


for $i=1, \ldots, \frac{n}{2}$, and

$$
\frac{\partial V_{i}}{\partial z_{i}^{2}} \geq 0 \Leftrightarrow \beta \leq \frac{n-2}{n}
$$

for $i=\frac{n}{2}+1, \ldots, n$. This means that, for all traders, sending a positive amount of the good initially owned starts to become interesting if, and only if, the condition $\beta \leq \frac{n-2}{n}$ is satisfied. Therefore the exchange economy defined above has the trivial equilibrium as unique oligopoly equilibrium whenever this condition is violated $\left(\frac{n-2}{n}<\beta \leq 1\right)$. Notice that when $n \rightarrow \infty$, the competitive allocation, which assigns the bundle $(0,1)$ (resp. $(1,0)$ ) to all agents $i=1, \ldots, \frac{n}{2}$ (resp. $\left.i=\frac{n}{2}+1, \ldots, n\right)$, must also become the outcome of an oligopoly equilibrium: namely, the one corresponding to the array of strategies $(1, \ldots, 1 ; 1, \ldots, 1)$ in which all agents supply the market with the total amount of their initial endowment.

It is not surprising that existence does not follow easily from very general assumptions on the economy. Even in partial analysis, the existence of a Cournot equilibrium cannot be established in full generality and requires a bundle of specific assumptions (see, for instance, Novshek, 1985). One should not be surprised that such specific assumptions have also to be formulated when one wishes to establish existence theorems for an oligopoly equilibrium in the general equilibrium framework. In fact, this constitutes an open field for future research in this area. The only positive result concerning the existence of an oligopoly equilibrium has been recently established by Bonnisseau and Florig, 2003, in the framework of linear exchange economies. By definition, an exchange economy is linear when the utility function of agent $i, i=1, \ldots, n$, is specified as

$$
u_{i}(x)=b_{i} \cdot x
$$

for some given vector $b_{i} \in R_{+}^{m} .{ }^{4}$ Given an $n$-tuple of strategies $\left(y_{1}, \ldots, y_{i}, \ldots, y_{n}\right)$, the maximisation problem referred to above takes the particular form

$$
\max _{x} b_{i .}\left(w_{i}-y_{i}+x\right) \quad \text { s.t. } \quad p . x \leq p . y_{i} \text { and } x \geq 0 .
$$

Then it follows from Gale, 1976, that there exists a non-empty set of equilibrium price vectors such that the $n$-tuple of solutions $x_{i}\left(p, y_{i}\right) i=1, \ldots, n$, to the above problems forms a competitive equilibrium with any of these price vectors in the exchange economy having the vectors $y_{i}$ as initial endowments. Even if the equilibrium price vector does not need to be unique, the corresponding utility levels $V_{i}\left(y_{i}, y_{-i}\right)$ are unique however, with

$$
V_{i}\left(y_{i}, y_{-i}\right)=b_{i} \cdot\left(w_{i}-y_{i}\right)+y_{i} \cdot \max _{j=1, \ldots, m}\left\{\frac{b_{i}^{j}}{p^{j}}\right\}
$$

for any price vector $p$ with which the vectors $x_{i}\left(p, y_{i}\right), i=1, \ldots, n$, define a competitive equilibrium. Finally, we introduce the notion of regular initial endowments, as defined by Bonnisseau and Florig, 1997, for the case of linear exchange economies. The initial endowments $w_{1}, \ldots, w_{i}, \ldots, w_{n}$ are regular when the corresponding equilibrium price vector $p$ is unique and, if for some competitive allocation $\left(x_{1}, \ldots, x_{i}, \ldots, x_{n}\right)$ and for all $j \in\left\{j: b_{i_{i j}} / p_{j}=\max _{k=1, \ldots, m}\left\{b_{i}^{k} / p^{k}\right\}\right\}$, we have $x_{i}^{j}>0$. The counterexample provided

\footnotetext{
${ }^{4}$ An example of a linear exchange economy is provided above with $b_{i}=(\beta, 1)$ for $i=1, \ldots, \frac{n}{2}$, and $b_{i}=(1, \beta)$ for $i=\frac{n}{2}+1, \ldots, n$.
} 
above involves regular initial endowments. Bonnisseau and Florig have shown that, if the initial endowments $w_{1}, \ldots, w_{i}, \ldots, w_{n}$ are regular, when a linear exchange economy, say $E_{1}$, is replicated $k$-times $\left(E_{k}\right)$, there exists an integer $k_{o}$ such that, for every $k \geq k_{o}$, the vector of strategies $\left(w_{1}, \ldots, w_{i}, \ldots, w_{n}\right)$ replicated $k$-times is an oligopoly equilibrium in the economy $E_{k}$. It is easy to see that the allocation

$$
\left(x_{1}\left(p\left(w_{1}, \ldots, w_{i}, \ldots, w_{n}\right), w_{1}\right), \ldots, x_{n}\left(p\left(w_{1}, \ldots, w_{i}, \ldots, w_{n}\right), w_{n}\right)\right)
$$

corresponding to this oligopoly equilibrium and the equilibrium price vector $p\left(w_{1}, \ldots, w_{i}\right.$, $\left.\ldots, w_{n}\right)$ form a competitive equilibrium in the economy $E_{1}$. Accordingly, this result shows that, if the linear exchange economy is finite, but sufficiently large, price-taking behaviour necessarily leads to an oligopoly equilibrium. The strength of this result follows from the fact that it is based on the "fundamentals" of the economy, and not on some untestable assumptions, like the assumption of strict quasi-concavity of the payoff functions, which is often used in partial analysis. The above proposition is best illustrated using our counterexample to the existence. As stated above, this counterexample satisfies the assumption of regular initial endowments. Assume that $\beta=\frac{3}{4}$ so that, when $n=4, \frac{n-2}{n}=\frac{1}{2}<\beta$. Consequently, the only oligopoly in the economy $E_{1}$ is the trivial equilibrium. Yet this ceases to be true in the economy $E_{2}$ consisting of four agents of each type since then we obtain $\frac{n-2}{n}=\frac{3}{4}=\beta$, so that the necessarily and sufficient condition for the array of strategies $((1,0), \ldots,(1,0) ;(0,1), \ldots,(0,1))$ to be an oligopoly equilibrium is now fulfilled.

To conclude this note, it is worth noticing that, using a replication procedure "à la Debreu-Scarf", Lahmandi-Ayed, 2001, has been able to prove that, under standard assumptions, a sequence of allocations corresponding to symmetric oligopoly equilibria in a replicated economy infinitely many times necessarily converges to a competitive allocation of the basic economy. Notice that this result guarantees the convergence of the price allocations corresponding to these oligopoly equilibria without ensuring the convergence of the strategies corresponding to these equilibria. Notice also that the convergence is proved only for symmetric oligopoly equilibria. In the case of asymmetric equilibria, the dimension of the price allocations space increases unboundedly as the economy is replicated, making it difficult to link these magnitudes to the basic exchange economy.

\section{References}

J. M. Bonnisseau and M. Florig (2003), Oligopoly equilibria in large, but finite, linear economies, Economic Theory, 22.

J. M. Bonnisseau and M. Florig (1997), Continuity and uniqueness of equilibria for linear exchange economies, Cahiers CERNSEM, Université de Paris I.

G. Codognato and J. Gabszewicz (1991), Economie de Cournot-Walras dans une économie d'échange, Revue Economique, 1013-1026.

T. Cordella and J. Gabszewicz (1998), "Nice" trivial equilibria in strategic market games, Games and Economic Behaviour 22, 162-169. 
A. Cournot A. (1838), Recherches sur les Principes Mathématiques de la Théorie des Richesses, Paris. Translation: Bacon, A. (1897), Researches into the Mathematical Principles of the Theory of Wealth, McMillan, New York.

G. Debreu and H. Scarf (1963), A limit theorem on the core of an economy, International Economic Review 4, 235-246.

P. Dubey and M. Shubik (1978), The non cooperative equilibria of a closed trading economy with market supply and bidding strategies, Journal of Economic Theory 17, 1-20.

J. Gabszewicz (2002), Strategic Multilateral Exchange, Edward Elgar Publishing, Cheltenham.

D. Gale (1976), The linear exchange model, Journal of Mathematical Economics 3, 205-209.

R. Lahmandi-Ayed (2001), Oligopoly equilibria in exchange economies: a limit theorem, Economic Theory 17, 665-674.

W. Novshek (1985), On the existence of a Cournot equilibrium, Review of Economic Studies 52, 85-98.

A. Wald (1936), On some systems of equations of mathematical economics, ZfN (translated, 1951, Econometrica). 